\title{
Vive l'Empereur!
}

Erhard Taverna

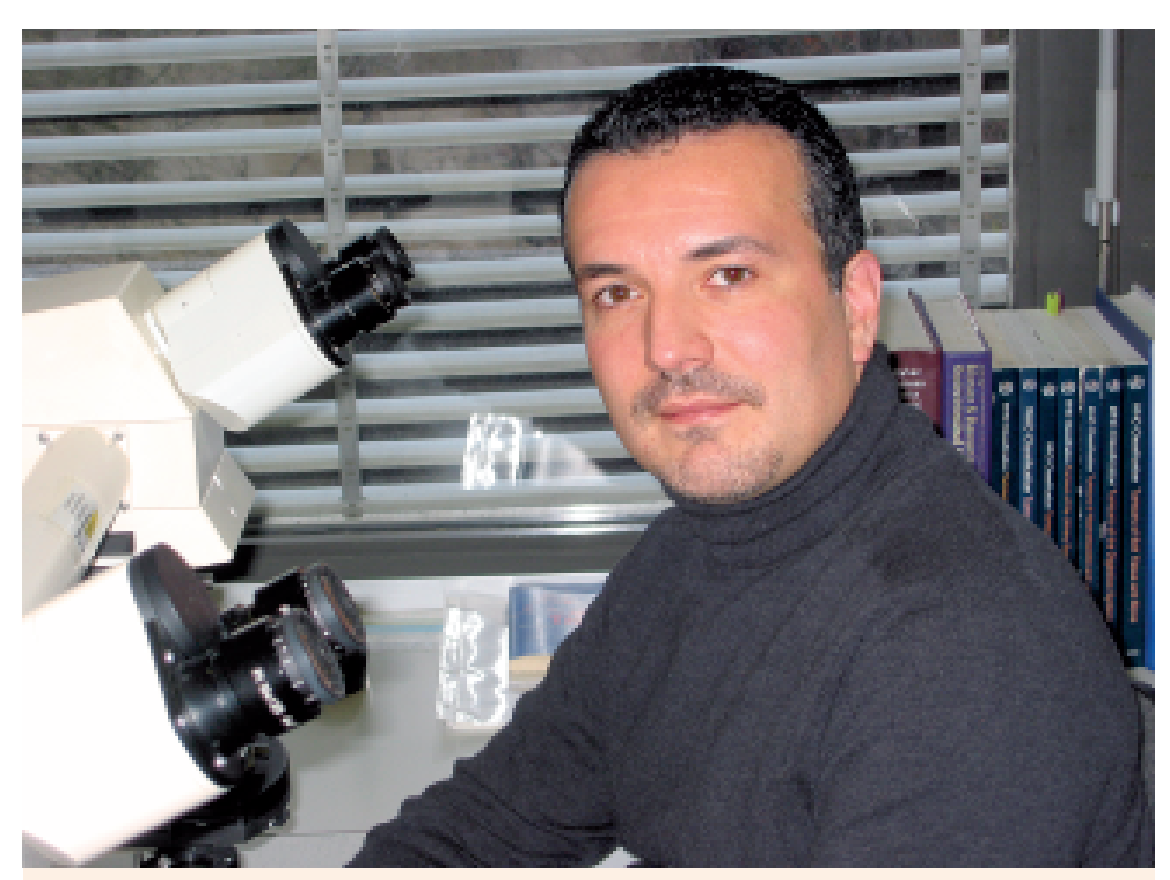

Dr. med. Alessandro Lugli, Institut für Pathologie, Universitätsspital Basel, Schönbeinstrasse 40, 4031 Basel, E-Mail: alugli@uhbs.ch.

Sechs Jahre nach seiner Ankunft auf der Insel St. Helena starb Napoleon Bonaparte am 5. Mai 1821 in britischer Gefangenschaft. Noch fast 200 Jahre später vermag der korsische General Anhänger und Gegner gegeneinander aufzubringen. Der weit abgelegene Exilort im Südatlantik und der erstmals 1961 nachgewiesene erhöhte Arsengehalt in verschiedenen Haarproben liess Legenden und Verschwörungstheorien kräftig tion von dieser Figur. Daraus geworden sind eine Privatbibliothek mit rund 1600 Bänden und zwei wissenschaftliche Arbeiten als Früchte von Hobby und Beruf. Die erste wurde im April 2005 in «Human Pathology» veröffentlicht, die zweite im Januar 2007 als Fallstudie in «Nature Clinical Practice». Der Oberarzt an der Pathologie des Universitätsspitals Basel hat sich auf Gastroenterologie spezialisiert und will sich nach einem Forschungsaufenthalt in Montreal ganz auf das Kolonkarzinom konzentrieren.

Napoleon verfügte, dass sein Leibarzt Dr. Francesco Antommarchi eine Autopsie durchführen sollte. Diese wurde nach seinem Ableben am 6. Mai, in Gegenwart von sieben englischen Ärzten, vorgenommen und sowohl in den Memoiren des Arztes wie auch in einem Bericht an den Sohn Napoleons, den «König von Rom», festgehalten. Der Bericht schildert exakt, was die Ärzte vorfanden: Ein sehr blasses Herz ohne Blutungen. Kaffeesatzähnliches Material im Magen, eine verhärtete, ulzerierte und unregelmässig begrenzte Wunde der Magenwand, von der Cardia bis zum Pylorus reichend. Zusätzlich ein präpylorisches, an der Leber haftendes Ulkus. Ein verdicktes kleines Bauchnetz und vergrösserte und verhärtete perigastrische Lymphknoten. Lugli vergleicht den historischen Autopsiebericht mit den makroskopischen Bildern von 50 histologisch gutartigen Ulzera und 50 Magenkarzinomen aller vier Tumorstadien. Antommarchis Beschreibung innerer Organe, von Tumorgrösse, Infiltrationstiefe und Beschaffenheit der Lymphknoten deckt sich mit den Befunden von 135 von 1986 bis 2003 behandelten Tumorpatienten. Die damalige Diagnose eines Magenkarzinoms mit finaler Massenblutung wird bestätigt, nach heutiger Nomenklatur handelte es sich um mindestens ein T3N1M0-Stadium IIIA. Selbst wenn Napoleon ein nochmaliger Fluchtversuch, wie zuvor von Elba, gelungen wäre, hätte er medizinisch gesehen keine Chancen gehabt, die Geschichte wieder umzuschreiben. Aufgrund der fehlenden Kachexie bestreiten die Anhänger der Vergiftungsthese die erwähnte Diagnose. Luglis erste Arbeit entkräftet den Vorbehalt mit einer eleganten Beweisführung. Dagegen sprechen die Dicke der gemessenen Bauchfettschicht und die gemessenen Bundweiten der kaiserlichen Hosen von 1800 bis 1821. Die lineare Relation von Bauchfett und BMI ergibt Spiel und mit ihm eine lebenslängliche Faszina- wuchern. Wer sich in das Labyrinth hineinwag Spiegelmagazin bis zum Fernse CNN. Auf dem Pult des Pathologen Dr. med. liegt ein dicker Stapel von Druckauszügen mit Kommentaren der ganzen Weltpresse.

Wie alle Neunjährigen interessierte sich A sandro Lugli für Soldaten. An Weihnachten und Modellbauschachteln Modell Airfix 1:72 m 48 Plastikfigürchen. Damit liessen sich Schlachten nachspielen und einmal war einer mit einem merkwürdigen Hut dabei. So kam der Kaiser ins 
einen Wert von $27,2 \mathrm{~kg} / \mathrm{m}^{2}$, entsprechend einem Gewicht von $75,9 \mathrm{~kg}$ (Kontrollgruppe BMI 12,4-30,6). Aus zwölf Hosenpaaren der Exilzeit errechnet der Autor ein Todesgewicht von 79,7 kg. Zwei Berechnungsmethoden, die den Arsenanhängern Magenschmerzen bereiten. Sie müssen auch damit leben, dass keine der obligaten Symptome einer chronischen Vergiftung wie Hornhautverdickungen der Hände und Füsse oder subendokardiale Blutungen im linken Ventrikel bekannt sind.

Arsen gab es damals in Kosmetika, im Wein damit behandelter Fässer, in Aufbewahrungsmitteln und Tapetenfarben. Positive Haarproben gehen schon auf die Zeit vor St. Helena zurück.
Doch die Gläubigen der Arsengemeinschaft lassen sich auch jetzt nicht beirren. Einer der führenden Anhänger hat sich schon gemeldet: «The ridiculous speculation of ignorant doctors.»

Im Foyer des Instituts an der Schönbeinstrasse 40 sind Merkblätter «Pathologie - Wozu?» aufgelegt. «Die Autopsie schafft Sicherheit über Todesursache und Erkrankung sowie begleitende Leiden», steht da geschrieben. Eine grosse Baumscheibe hängt daneben an der Wand. Zwei Jahre nach dem Friedensschluss zwischen Preussen und Frankreich von 1795 in Basel liess der Korse hier eine Platane pflanzen. Es ist nicht leicht, aus seinem Schatten zu treten. 